\title{
NEXT GENERATION SEQUENCING IDENTIFIED A NOVEL MULTI EXON DELETION OF THE NF1 GENE IN A CHINESE PEDIGREE WITH NEUROFIBROMATOSIS TYPE 1
}

\author{
Yang $\mathrm{J}^{1, \#}$, An J-X ${ }^{1, \#}$, Liu X-L ${ }^{1}$, Wang Z-Q ${ }^{1}$, Xie G-M ${ }^{1}$, Yang X-L ${ }^{2}$, Xu S-J ${ }^{1}$, Feng F ${ }^{1}$, Ni $\mathrm{Y}^{1, *}$ \\ \# Jie Yang and Jinxia An contributed equally to this study. \\ *Corresponding Author: Dr. Yali Ni, Gansu Provincial Maternity and Child-Care Hospital, 143 Qilihe \\ North Street, LanZhou, Gansu Province, 730050, People's Republic of China. Tel.: +86-0931-2231241. \\ E-mail: niyali@126.com
}

\section{ABSTRACT}

Neurofibromatosis type 1 (NF1) is a genetic disease involving neurocutaneous abnormalities. Neurofibromatosis type 1 is an autosomal dominant disorder characterized by the neurofibromas and café-au-lait spots. Mutation in the NF1 gene causes NF1. The NF1 gene encodes neurofibromin. In this study, we found a 31-year-old Chinese boy with NF1. He presented only with café-au-lait spots over the whole body. The proband's mother had a severe phenotype with neurofibroma and café-au-lait macules over her whole body, mostly in the facial region. A novel multi exon deletion c.(4661+1_4662-1)_(5748+1_5749-1)del; [EX36_39DEL] on the $N F 1$ gene has been identified in the proband. Quantitative real-time polymerase chain reaction (qPCR) confirmed that this mutation is co-segregated well and was inherited from the proband's mother. The mutation was absent in the proband's father and normal individuals. The novel multi exon deletion results in the formation of a truncated NF1 protein that caused the NF1 phenotype in this family. Our present study also emphasized the significance of rapid, accurate and cost-effective screening for the patient with NF1 by next generation sequencing (NGS).

Keywords: Café-au-lai-spots; Multi-exon deletion; Novel mutation; Neurofibromas; Neurofibromatosis type 1 (NF1); Next generation sequencing (NGS).

\footnotetext{
${ }^{1}$ Gansu Provincial Maternity and Child-Care Hospital, LanZhou, Gansu Province, People's Republic of China.

2 The third people's hospital of Liangzhou District, Wuwei City, Gansu Province, People's Republic of China.
}

\section{INTRODUCTION}

Neurofibromatosis type 1 (NF1) [MIM\# 162200] is a rare, autosomal dominant neurocutaneous disease. Neuro-fibromatosis type 1 manifests with neurofibroma (fibromatous skin tumors), café-au-lait spots and skin fold freckling [1]. Neurofibromatosis type 1 patients usually present with extreme inter- and intra-familial phenotypic variability with an incidence of 1/3500 live births [1]. Germline mutations in the NF1 gene causes NF1. The NF1 gene encodes neurofibromin consisting of 2818 amino acids, highly expressed in neurons and different types of neuronal cells. In addition, neurofibromin activates protein by down-regulating the function of the renin-angiotensin system (RAS) [1].

\section{CASE REPORTS}

Two individuals in a Chinese family were clinically diagnosed with NF1, based on the consensus criteria of the National Institutes of Health (NIH) [2], at the Gansu Provincial Maternity and Child-Care Hospital, LanZhou, Gansu Province, People's Republic of China (PRC) (Figure 1). In our hospital, detailed physical examination, laboratory investigations were done. The proband (II-1) was a 31-year-old Chinese male with neurofibroma and café-au-lait macules on his chest and back [Figure 2(A), 2(B)]. In the proband, café-au-lait macules are gradually increasing in size as well as developing new café-au-lait macules. Several tender masses were identified on the chest and back of the proband. Physical examination of these tender masses were consistent with neurofibroma. 


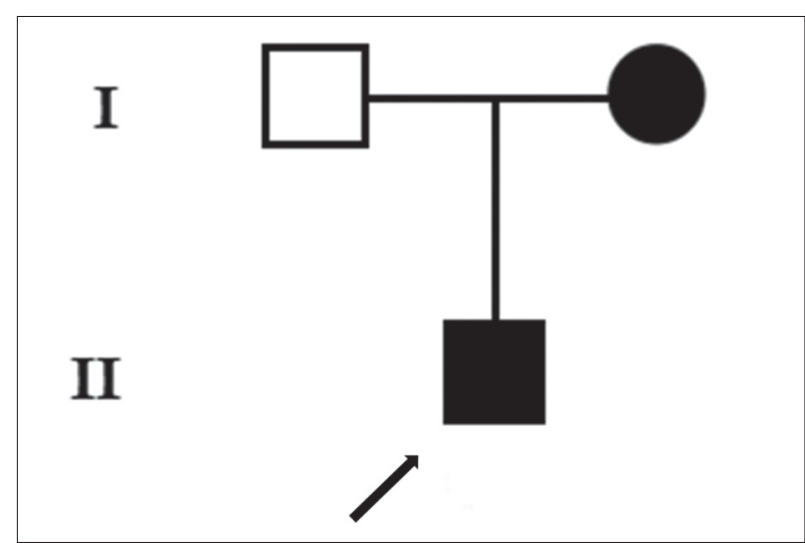

Figure 1. Pedigree of this Chinese family. The Chinese family is co-segregating with the NF1 mutation. Squares and circles indicate males and females, respectively; open symbols indicate unaffected individuals, filled symbols indicate affected individuals; arrows indicate the proband in this case.

The proband's mother (I-2) was 65 years old and presented with multiple subcutaneous neurofibromas and café-au-lait spots [Figure 2(C), 2(D) and 2(E)]. The proband's father (I-1) was normal. Physical growth and cognitive ability was normal and no other abnormality was identified in either the proband or his mother.

The Ethics Committee of the Gansu Provincial Maternity and Child-Care Hospital approved the present study. Next generation sequencing (NGS) and Sanger sequencing was performed to identify the candidate gene and mutation in the proband. The proband's blood sample was collected and genomic DNA was extracted.

Next Generation Sequencing. DNA samples obtained from the proband (II-1) were sequenced using target exome-based NGS. A sequence capture human array (Roche NimbleGen Inc., Madison, WI, USA) was designed to capture the targeted sequence, covering all 57 exons and flanking sequences (including the $100 \mathrm{bp}$ introns) of the $N F 1$ genes that are associated with NF1. On average, there are $6,857,687$ reads per sample, with $87.48 \%$ of mapping to the targeted regions. The average sequencing depth of the target area was 428.14 -times with $99.86 \%$ coverage.
The procedure for preparation of libraries was consistent with standard operating protocols published previously [3]. According to the standard protocol, we simultaneously sequenced 30 samples on Illumina HiSeq 2500 Analyzers (Illumina, San Diego, CA, USA) for each pooling batch of 90 cycles (specially designed rare disease screening). We applied Illumina Pipeline software (version 1.3.4) to generate the raw data that was later used for bioinformatic analysis. We extracted the clean reads from the raw reads by using already established filtering criteria. We then selectively used at least $90 \mathrm{bp}$ long clean reads for aligning to the human reference genome (Build 37) of the National Center for Biotechnology Information (NCBI) (https:// www.ncbi.nlm.nih.gov/grc) database using Burrows Wheeler aligner (BWA). The BWA is a multi-vision software package (http://bio-bwa.sourceforge.net/index. shtml) generating the output file in BAM format. After that, we analyzed the target region coverage, sequencing depth, SNP/InDel/CNV detection by using the bam data. Later, SOAPsnp software (www.soap/genomics.org.cn), Sam tools pileup software (www.htslib.org) and Bioinformatic computational framework (this explains the pipeline made by the software), were established for identifying single nucleotide polymorphisms (SNPs), insertions/deletions (InsDels) and copy number variations (CNVs). Filtering criteria for a SNP or InDel has been set with at least 10 reads with $>20.0 \%$ of total reads. The SNPs are filtered out if it's frequency is $<0.05$ in dbSNP (www.ncbi.nlm. nih. gov/projects/SNP/), Hapmap (https://www.genome. gov), 1000 Genomes Project (http://www.1000genomes. org), the 200 healthy reference samples sequenced in this study, it would consider as a disease-causing mutation.

\section{Identification of a Novel Heterozygous Multi Exon}

Deletion in the NF1 Gene. Next generation sequencing identified a novel multi exon c.(4661+1_4662-1)_(5748 +1_5749-1)del; EX36_39DEL deletion on the NF1 gene in the proband and in his mother. This multi-exon deletion leads to the formation of a truncated neurofibromin instead of the wild type neurofibromin. This mutation has been validated in proband and his parents by q-PCR
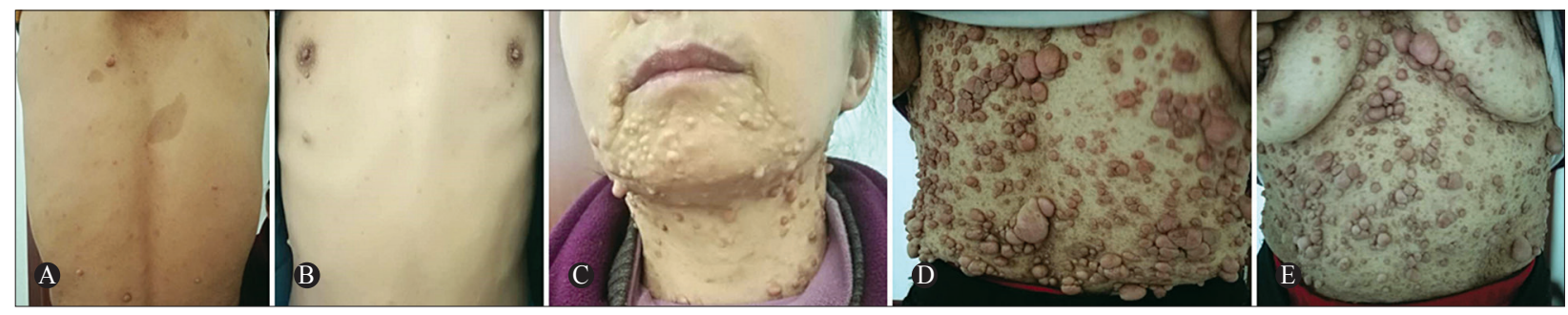

Figure 2. Clinical descriptions. Shows several neurofibromas and café-au-lait macules on the proband's (II-1) chest (A) and back (B). Shows many neurofibromas and café-au-lait macules on the proband's mother's face (C), back (D) and chest (E). 


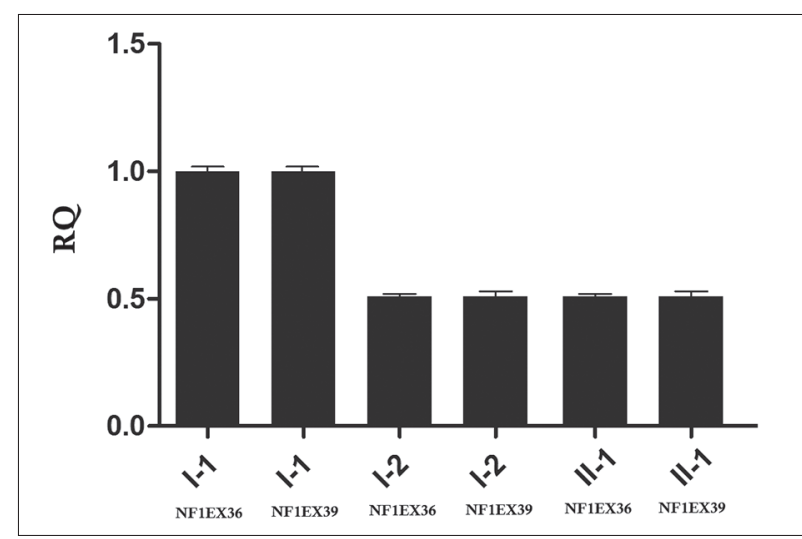

Figure 3. Mutation screening. Mutational analysis (NGS and q-PCR) of the NF1 gene in this family. Next generation sequencing result was validated by qPCR in the proband (II-1), his father (I-1) and mother (I-2) (GenBank Accession: NM_000267.3).

(Figure 3). This mutation has not been identified among the 100 normal healthy control subjects.

Quantitative Real-Time Polymerase Chain Reaction (qPCR). We performed qPCR to validate the multi exon deletion on the NF1 gene identified by NGS. The qPCR was performed using an ABI PRISM ${ }^{2} 7900 \mathrm{HT}$ realtime PCR system (Life Technologies, Carlsbad, CA, USA) and hot-start qPCR Master Mix. The primer sequences and PCR conditions will be provided by the corresponding author upon request. Data were analyzed using the comparative threshold cycle $\left(2^{-\Delta \Delta \mathrm{Ct}}\right)$ method.

\section{DISCUSSION}

Neurofibromatosis type 1 is a rare neurocutaneous inherited genetic disease caused by germline mutations in the $N F 1$ gene. In the human genome, $N F 1$ is one of the largest genes with a very high mutation rate with extreme mutational heterogeneity. Over 1000 germline (missense, deletion, insertion, nonsense, splice site mutations) mutations of the NF1 gene have been reported so far [4]. In this study, the novel heterozygous multi exon deletion causes the formation of a truncated neurofibromin which affects the normal function of the NF1 gene. Neurofibromatosis type 1 patients usually presented with two major clinical symptoms; i.e., neurofibromata and the café-au-lait spots, which increase in number and gradually developed into larger spots with increasing age. Hence, a clear gradient or degree of phenotypic variation is common in affected individuals of the same family [5]. Recently, in their two studies, Banerjee et al. [1,6] reported that clinical manifestations for NF1 patients are extremely heterogenous, even associated with astrocytoma, tibial pseudarthrosis and anemia. In this study, we showed that the proband and his mother presented with severe and mild phenotypes, respectively, although they carry the same mutation. Moreover, in addition of the genetic factors, there are also epigenetic factors that influence the phenotypic heterogeneity in NF1 patients [1].

In conclusion, we identified a Chinese family with NF1. Next generation sequencing and qPCR identified a novel heterozygous multi exon deletion c. $(4661+1$ 46621)_(5748+1_5749-1)del; (EX36_39DEL) on the NF1 gene causing NF1 in this family. We have added a heterozygous novel NF1 mutation associated with an NF1 phenotype as well as understanding the signification of the application of NGS for rapid and accurate screening of the specific disease-associated genes that provide the correct clinical diagnosis for NF1.

\section{ACKNOWLEDGMENTS}

We wish to thank all the members of this family for participating in our study. We also thank Dr. Santasree Banerjee (Beijing Genomics Institute-Qingdao, Beijing Genomics Institute-Shenzen, Qingdao, Shandong Province, PRC) for her careful reading and editing of the manuscript.

This project was supported by Grant from The Gansu Natural Science Foundation of China (Grant Number: 17JR5RA027).

Declaration of Interest. The authors report no conflicts of interest. The authors alone are responsible for the content and writing of this article.

\section{REFERENCES}

1. Banerjee S, Dai Y, Liang S, Chen H, Wang Y, Tang $\mathrm{L}$, et al. A novel mutation in NF1 is associated with diverse intra-familial phenotypic variation and astrocytoma in a Chinese family. J Clin Neurosci. 2016; 31(9): 182-184.

2. Stumpf DA, Alksne JF, Annegers JF. Neurofibromatosis. Conference statement. Presented at the National Institutes of Health Consensus Development Conference, held at the National Institutes of Health in Bethesda, MD, USA on July 13-15 1987. Arch Neurol 1998;45(5): 575-578.

3. Yang L, Banerjee S, Cao J, Bai X, Peng Z, Chen H, et al. Compound heterozygous variants in the coiledcoil domain containing 40 gene in a Chinese family with primary ciliary dyskinesia cause extreme phe- 
notypic diversity in cilia ultrastructure. Front Genet. 2018; 9(2): 23. doi: 10.3389/fgene.2018.00023. eCollection 2018.

4. Pasmant E, Parfait B, Luscan A, Goussard P, BriandSuleau A, Laaaaurendeau I, et al. Neurofibromatosis type 1 molecular diagnosis: What can NGS do for you when you have a large gene with loss of function mutations? Eur J Hum Genet 2015; 23(5): 596-601.
5. Boyd KP, Korf BR, Theos A. Neurofibromatosis type 1. J Am Acad Dermatol. 2009; 61(1): 1-14.

6. Banerjee S, Lei D, Liang S, Yang L, Liu S, Wei Z, et al. Novel phenotypes of NF1 patients from unrelated Chinese families with tibial pseudarthrosis and anemia. Oncotarget. 2017; 8(24): 39695-39702. 